\title{
Not all mismatches are equal: importance of alloreactivity direction
}

\author{
Jacinta Perram ${ }^{1,2}$ and Nada Hamad ${ }^{1,2,3}$
}

${ }^{1}$ Department of Haematology, St Vincent's Hospital Sydney; ${ }^{2}$ St Vincent's Clinical School Sydney, University of New South Wales and ${ }^{3}$ School of Medicine Sydney, University of Notre Dame Australia, Sydney, Australia

E-mail: NADA HAMAD - nada.hamad@swha.org.au

doi:10.3324/haematol.2021.279587

\begin{abstract}
A llogeneic hematopoietic stem cell transplantation (HSCT) remains the only curative therapy for numerous hematologic malignant and benign conditions. Transplantation does, however, come with significant risks of morbidity and mortality related to the transplant, graft-versus-host disease (GVHD) and relapse. The art of medicine in allogeneic HSCT lies in walking the tightrope between relapse risk and GVHD. We have come a long way in minimizing the risks of GVHD with rates of clinically significant (grade 2-4) acute GVHD and moderate to severe chronic GVHD reported to be as low as $50 \%$ and $30 \%$, respectively. ${ }^{1,2}$ This is, no doubt, related to more widespread use of T-cell depletion methods and better HLA typing. ${ }^{3}$ Optimal donor selection in the absence of a matched relative relies on an assessment of the relative risk, and selection of the donor most genetically suitable based on HLA matching at HLA-A, -B, -C, DR and DQ. Human-leukocyte-antigen DPB1 (HLA-DPB1) mismatch is known to be broadly associated with decreased relapse at the cost of increased rates of acute GVHD. ${ }^{4}$ HLA-DPB1 mismatch in otherwise matched donors is common, yet our ability to predict GVHD severity based on this mismatch is limited. In this issue, Zou et al. ${ }^{5}$ present data supporting HLA-DPB1 mismatch associated risks of acute GVHD while using clinical correlation to investigate the clinical impact of HLA-DPB1 molecular mismatch.

In the last two decades, donor selection algorithms have classified HLA-DPB1 mismatches as permissive or nonpermissive, based on functional toxicity assays and T-cell
\end{abstract}

epitope (TCE) analysis. ${ }^{6,7}$ While these methods assess the qualitative character of a mismatch, they do not evaluate direction or anticipate immunogenicity of a given mismatch. This poorly characterized potential risk represents a limitation of current donor selection algorithms.

By contrast, molecular matching techniques assess structural components of epitopes, called eplets, allowing quantification of donor and recipient mismatched eplets (ME). This quantification, when combined with the PIRCHE score (PS), a predictor of TCE alloreactivity, has been shown to predict immunogenicity and clinical outcomes in haploidentical transplant recipients. ${ }^{8}$ Zou et al. ${ }^{5}$ present novel data on the use of molecular algorithms for HLA-DPB1 mismatch in a cohort of more than 1,500 patients who received an unrelated donor transplant between 2005-2018 at The University of Texas MD Anderson Cancer Center. The primary question in this study is whether molecular matching offers superior prognostic guidance than the traditional TCE model. The group reports concordance testing of bidirectional $\mathrm{ME}$ and PS, as well as the TCE model, with acute GVHD outcomes. The central finding is that high levels of $\mathrm{ME}$ in the graft-versus-host $(\mathrm{GVH})$ direction are the strongest single predictor of acute GVHD. The authors propose use of molecular algorithms to guide the choice of or augmentation of acute GVHD prophylaxis.

Another crucial finding is the importance of direction of alloreactivity. Bidirectional high ME or PS mismatch is universally associated with high rates of acute GVHD and

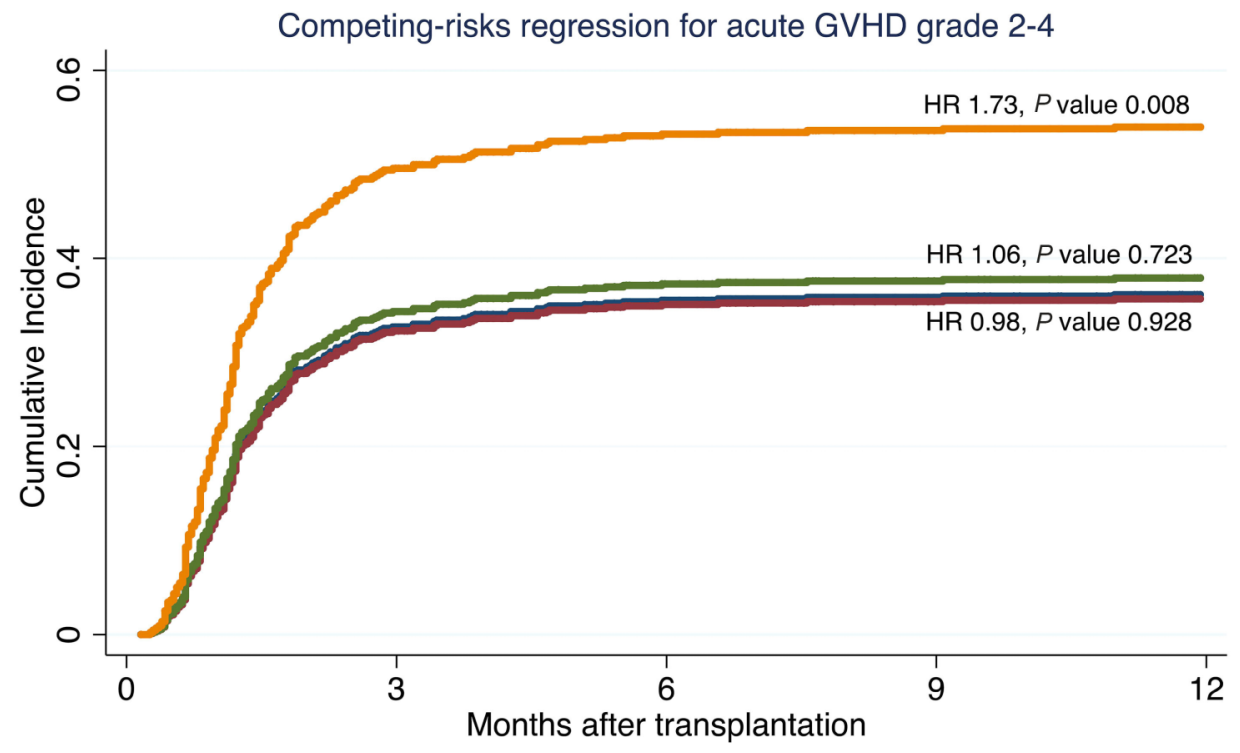

Figure 1. Competing risks regression for acute graftversus-host disease grade 2-4. GVHD: graft-versus-host disease; HR: hazard ratio; ME: mismatched eplets; GVH: graft-versus-host; HVG: host-versus-graft. 


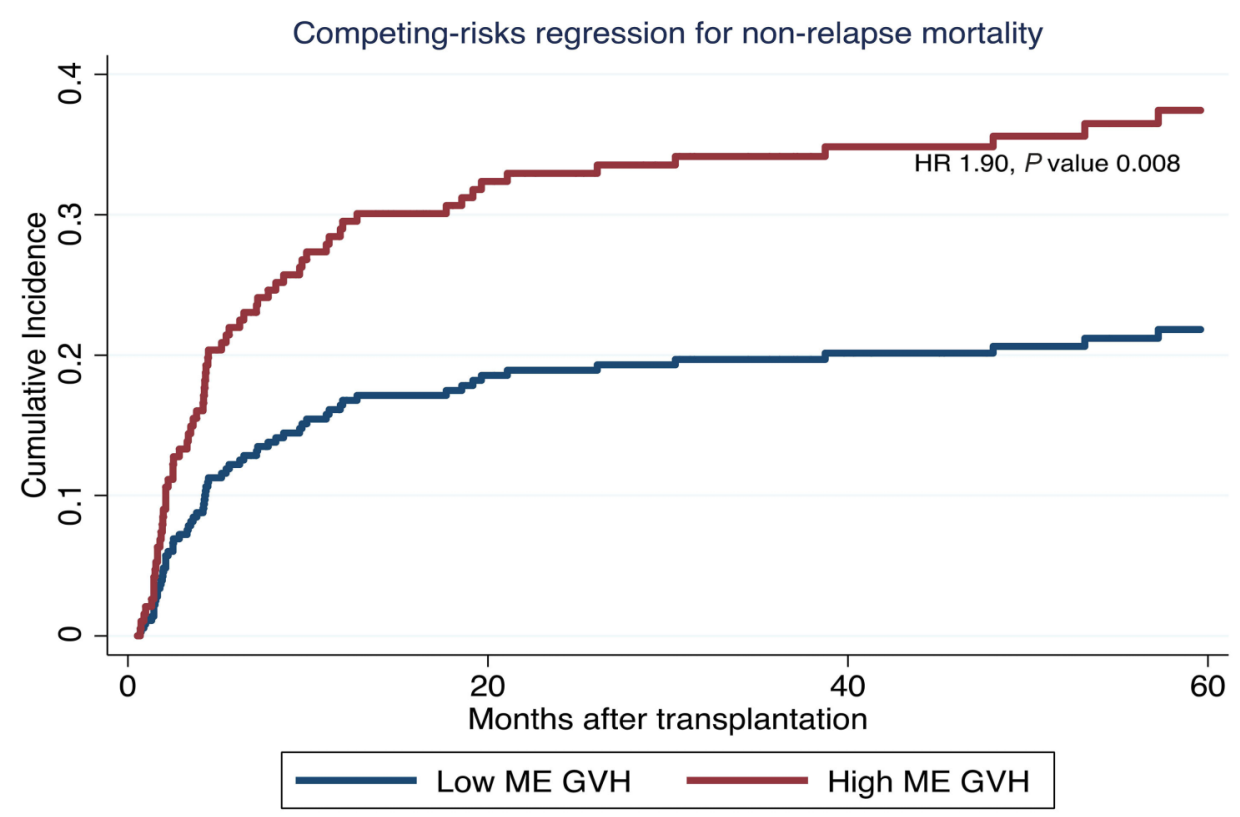

Figure 2. Competing risks regression for non-relapse mortality. HR: hazard ratio; ME: mismatched eplets; GVH: graft-versus-host.

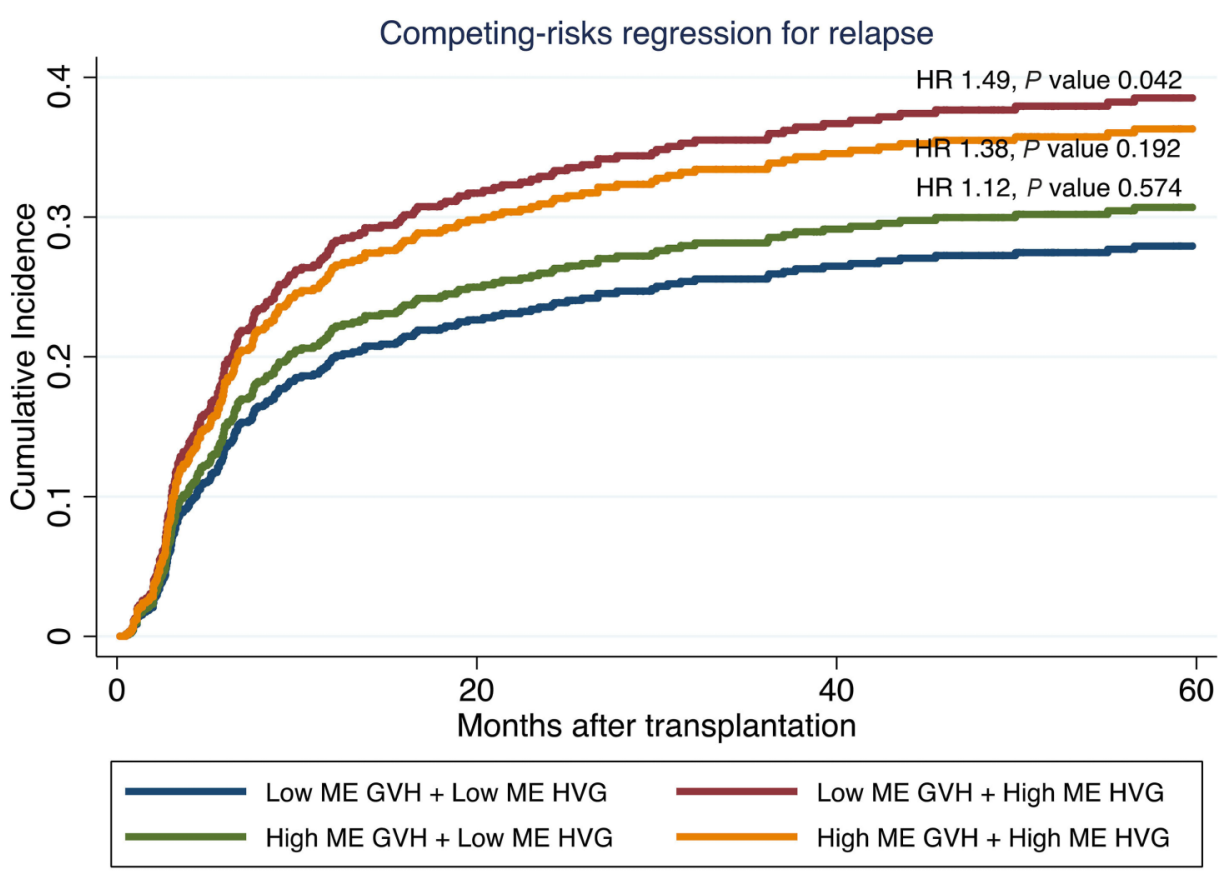

Figure 3. Competing risks regression for relapse. HR: hazard ratio; ME: mismatched eplets; GVH: graft-versus-host; HVG: host-versus-graft.

relapse, suggesting a synergistic effect. The reduced relapse risk purported to arise from HLA-DPB1 permissive mismatch was only observed among those with high ME or PS in the GVH direction, and not those with isolated high $\mathrm{PE}$ or MS in the host-versus-graft (HVG) direction, who in fact had an increased rate of relapse. This is a clinically important outcome as it forces re-evaluation of the rationale for tolerating increased GVHD in recipients of transplants with HLA-DPB1 permissive mismatch.

Building on the existing TCE model for HLA-DPB1 mismatch classification, there are several findings offering refined stratification. Among the permissive mismatch group, high HVG ME and PS are associated with a high risk of GVHD, yet among non-permissive mismatched cases, an isolated high HVG ME is associated with low GVHD risk. The empirical inconsistency of these results highlights the persisting incomplete understanding of HVG pathophysiology.

While the outcomes reported here have potential to alter practice in the future, the authors acknowledge the need for significant further study. Involvement of only a single center is a significant limitation. The retrospective nature of this research is not problematic in itself given the correlative nature of the analyses performed. However, one of the great challenges in allogeneic HSCT is the rapidly evolving landscape across which research is performed. Since the commencement of this study, the adoption of T-cell-depleting therapies has rapidly expanded. 
Across the trial period, the use of anti-thymocyte globulin increased significantly $(23.7 \%$ to $41.2 \%)$. It is unknown whether, and if so how, this has augmented results. Confirmatory investigations will be important to validate current findings and confirm them as enduring in the setting of routine T-cell depletion.

Another important development has been the use of post-transplant cyclophosphamide in haploidentical allogeneic HSCT. The associated rapid improvement in clinical outcomes has shifted the donor selection paradigm. If anything, the uptake of haploidentical transplants reinforces the importance of this trial. With increased availability of alternative donors, the imperative to refine outcome prediction in HLA-DPB1 mismatch is all the more relevant.

A further potentially significant development is related to the evolution of therapies for GVHD.,9 More efficacious treatment and prevention strategies for GVHD may redefine donor selection algorithms, permitting mismatches that were previously prohibited.

Zou et al..$^{5}$ present an important novel approach to assessment of HLA-DPB1 mismatch permissibility. The authors acknowledge that confirmation of their findings with multi-center data is needed before refinement of algorithms can be considered. One of the great challenges for the field of HSCT moving forward is access to progressively more specialized molecular testing. There is also the need to embrace international research collaborations to allow realtime outcome reporting in a rapidly progressing field.

\section{Disclosures}

No conflicts of interest to disclose.

\section{Contributions}

$J P$ and $N H$ wrote and edited the editorial.

\section{References}

1. Jagasia M, Perales MA, Schroeder M, et al. Ruxolitinib for the treatment of steroid-refractory acute GVHD (REACH1): a multicenter, open-label phase 2 trial. Blood. 2020;135(20):1739-1749.

2.Zeiser R, Polverelli N, Ram R, et al. Ruxolitinib for glucocorticoidrefractory chronic graft-versus-host disease. $N$ Engl J Med. 2021;385(3):228-238

3. Bacigalupo A. ATG in allogeneic stem cell transplantation: standard of care in 2017? Point. Blood Adv. 2017;1(9):569-572.

4. Fleischhauer K, Shaw BE, Gooley T, et al. Effect of T-cell-epitope matching at HLA-DPB1 in recipients of unrelated-donor haemopoieticcell transplantation: a retrospective study. Lancet Oncol. 2012;13(4):366-374

5. Zou J, Kongtim P, Oran B, et al. Refined HLA-DPB1 mismatch with molecular algorithms predicts outcomes in hematopoietic stem cell transplantation. Haematologica. 2022;107(4):844-866.

6.Zino E, Frumento G, Marktel S, et al. A T-cell epitope encoded by a subset of HLA-DPB1 alleles determines nonpermissive mismatches for hematologic stem cell transplantation. Blood, 2004;103(4):1417-1424.

7. Crivello P, Zito L, Sizzano F, et al. The impact of amino acid variability on alloreactivity defines a functional distance predictive of permissive HLA-DPB1 mismatches in hematopoietic stem cell transplantation. Biol Blood Marrow Transplant. 2015;21(2):233-241.

8. Zou J, Ciurea S, Kongtim P, et al. Molecular dispartiy in human leukocyte antigens is associated with outcomes in haploidentical stem cell transplantation. Blood Adv. 2020;4(15):3474-3485.

9. Zeiser R, von Bubnoff N, Butler J, et al. Ruxolitinib for glucocorticoirrefractory acute graft-versus-host disease. $N$ Engl J Med. 2020;382(19):1800-1810. 